Case Reports

\title{
Varicella Zoster Virus Causing Aseptic Meningitis Without Fever or Rash in an Immunocompetent Patient
}

\author{
Fazal I Raziq \\ Internal Medicine, Michigan State University, United States
}

Article history

Received: 03-04-2020

Revised: 24-04-2020

Accepted: 28-04-2020

Email: iraziq.fazal462@gmail.com

\begin{abstract}
Varicella-Zoster Virus (VZV) is known to be a cause of aseptic meningitis and can be potentially fatal. VZV meningitis can arise in immunodeficient and more rarely, immunocompetent hosts. We present here a rare case of VZV causing meningitis in a 44-year-old immunocompetent man. He presented with an isolated headache without any fever or rash. CSF PCR for VZV DNA was positive confirming VZV as the cause of meningitis. He was treated with IV ganciclovir for 3 days and was transitioned to oral valacyclovir to complete a total of 10 days of antiviral treatment with the resolution of the headache. The purpose of this case report is to highlight VZV as a cause of aseptic meningitis in immunocompetent individuals and emphasize the importance of using airborne precautions along with contact precautions in health care workers who deal with patients with VZV shingles.
\end{abstract}

Keywords: VZV, Meningitis, Immunocompetent

\section{Introduction}

\section{Background}

Varicella-Zoster Virus (VZV) is a common pathogen causing infection in the general population. Approximately 1.2 million people get infected by VZV annually in the united states. Primary infection presents as a vesicular rash with associated fever and pain (chickenpox). Secondary infection commonly occurs from virus reactivation in dorsal root ganglion when a patient becomes immunocompromised. Shingles is a common presentation of secondary infection from viral reactivation. Yawn et al. (2007) Rarely VZV causes aseptic meningitis with fever, rash and a headache in immunocompromised individuals.

\section{Case Presentation}

A 44-year-old male presents to the emergency department after three days of a right-sided headache with associated photophobia and phonophobia, body pains, nausea and emesis. The patient denied any recent travel, any associated mental status changes or focal neurological deficits, changes in vision, rash, any history of seizures or aura. The headache was described as sharp, became worse with movement and located in the frontal, temporal and occipital regions. He rated the headache as a 5/10 in intensity. Medical history included hypertension, first-degree atrioventricular block, Urolithiasis, asthma, gastrointestinal reflux disease and a remote history of smoking for 2 years. The patient was on albuterol inhaler for asthma and lisinopril for blood pressure control. The patient was seen the day prior to admission at urgent care for his headache and was given oral and inhaled steroids for nasal congestion and ibuprofen for his headache. He denied any improvement with prescribed medication. He reported, he came into contact with a child who had a fever, runny nose and cough four days prior to the presentation. He was up to date on immunizations; however, he was unsure if he was ever vaccinated against Varicella-Zoster Virus (VZV).

On presentation, the patient's temperature was $98.1^{\circ} \mathrm{F}$ [97.8-99. $\left.1^{\circ} \mathrm{F}\right]$, heart rate $109 / \mathrm{min}[60-100 / \mathrm{min}]$, respiratory rate $18 / \mathrm{min}[12-18 / \mathrm{min}]$, blood pressure 158/81 mmHg [90/60-120/80 mmHg], $\mathrm{SpO}_{2}$ 98\% [9599\%] on room air. He appeared ill, had chills and a subjective feeling of being warm. On examination, he was alert and oriented with no focal neurologic deficits. Deep tendon reflexes were normal. Motor examination and power were normal in all extremities. He had no nuchal rigidity, however, there was some occipital pain with passive and active range of motion with negative Brudzinski and Kernig signs. Tympanic membranes were normal and there were no abnormalities noted in the ear canal. No skin lesions were appreciated elsewhere. He had a normal cardiovascular, pulmonary and abdominal exam. 


\section{Investigations}

Laboratory results showed white blood cell count was within normal limits at $810 * 3 / \mathrm{uL}[4.0-12.010 * 3 / \mathrm{uL}]$ with elevation in neutrophil percentage at $83.3 \%$ [49.0$81.0 \%]$, decreased lymphocyte percentage at 12.8 [14.0$41.0 \%$ ], hematocrit decreased at 40.1 [42.0-49.5\%]. Comprehensive metabolic panel results included glucose $140[65-99 \mathrm{mg} / \mathrm{dL}]$, alanine aminotransferase elevated at $58 \quad[3-45 \mathrm{U} / \mathrm{L}]$. Urine analysis, thyroid-stimulating hormone, C-reactive protein, troponin, magnesium, activated partial thromboplastin time, prothrombin time were all within normal limits. HIV testing was negative. Electrocardiogram (ECG) showed tachycardia at 100/min and PR prolongation; the PR prolongation was consistent with the patient's prior ECG from 3 years prior. Computed tomography of the head and chest radiograph were normal. Lumbar Puncture (LP) was performed and it showed an intracranial pressure of 60 mmHg. Cerebrospinal Fluid Showed (CSF) an abnormal hazy appearance [clear], glucose elevated at 77 [40-70 $\mathrm{mg} / \mathrm{dL}]$, protein elevated at $105[15-45 \mathrm{mg} / \mathrm{dL}]$, red blood cell 4 [0-5], white blood cell elevated at 864 [0-5] with lymphocytic pleocytosis, lactate $2.7 \mathrm{mmol} / \mathrm{L}$ [0-2.8 $\mathrm{mmol} / \mathrm{L}]$. The blood glucose level prior to Lumbar puncture was 170 . The CSF to blood glucose ratio was 0.45. A Polymerase Chain Reaction (PCR) CSF panel was sent for analysis that included detection of the following organisms: Herpes Simplex Virus I and II, Human Herpes Virus-6, Parechovirus, Cytomegalovirus (CMV), Adenovirus, Escherichia coli $\mathrm{K} 1$ strain, $\mathrm{H}$. influenza, Listeria monocytogenes, Neisseria meningitidis, Streptococcus agalactiae, Streptococcus pneumoniae, Parainfluenza, Metapneumovirus, Cryptococcus neoformans, Cryptococcus gattii, Enterovirus and VZV. Blood cultures were drawn.

\section{Treatment}

The patient was started on intravenous Vancomycin, Ceftriaxone and Ganciclovir. There was a national shortage of intravenous Acyclovir in the united states at that time. Hence Ganciclovir was used instead.

Infectious Disease consultation was obtained, who recommended that the patient be continued on Intravenous (IV) Vancomycin, Ceftriaxone and Ganciclovir. The patient was also given IV isotonic fluids, antiemetics and pain medication for the headache. $\mathrm{He}$ was admitted and put under hospitalist care.

On day 2, the patient continued to have the same level of headache despite pain medication. There was no improvement in the patient's headache even after the lumbar puncture arguing against increased intracranial pressure as the cause of the headache. He remained afebrile. The neurologic exam continued to be normal.
No skin lesions were appreciated, including inside the ear canal. Nasopharyngeal swab for adenovirus and metapneumovirus by PCR were submitted for analysis. CMV DNA levels in blood were measured by PCR along with IgM and $\mathrm{IgG}$ antibodies to CMV; these were all negative.

On hospital day 3 , the patient's headache improved, and he had less fatigue. The CSF meningitis panel returned for a positive result for VZV by PCR. He also had VZV DNA PCR by blood drawn to rule out viremia and it was negative. IV Vancomycin and IV Ceftriaxone were stopped, and the patient was continued on IV Ganciclovir.

On day 4, the patient's headache and other symptoms had resolved, and he was discharged in stable condition. He was recommended to complete a course of oral Valacyclovir to complete a total of 10 days of antiviral therapy.

\section{Follow Up}

He was seen 2 weeks later in the clinic with no neurological sequelae.

\section{Discussion}

Varicella-Zoster Virus (VZV), a member of the Herpes virus family is a common pathogen infecting humans. In the united states, approximately, 1.2 million individuals get infected by the VZV virus annually. Yawn et al. (2007) Incidence is directly related to depressed cellmediated immunity. Weinberg et al. (2010; Miller, 1980) Thus the risk factors associated with the disease are those which depress the cell-mediated immune response including age, HIV-infected patients, transplant patients and patients with autoimmune diseases receiving specific immunomodulatory therapies, cancer patients on chemotherapy and patients on chronic steroid therapy. Harpaz et al. (2008; Chen et al., 2014; Antonelli et al., 1991) Other risk factors with poorly understood mechanism but significant evidence of association includes female sex, white ethnicity and physical trauma. Opstelten et al. (2006; Schmader et al., 1995; Thomas et al., 2004). In our case, there was no risk factor suggesting a depressed cell-mediated immunity.

VZV is usually transmitted by direct contact or airborne transmission from a patient infected with VZV with active herpes zoster lesions to a patient who is VZV-naïve (i.e., a person who has never had varicella or varicella vaccine). Josephson and Gombert (1988; Yoshikawa et al., 2001) Our patient had no symptoms suggestive of VZV infection in the past.

The mechanism of infection is thoroughly described in the literature. The VZV virus infects the cytotoxic T cells and then spreads to the regional lymph nodes. Ultimately infecting the epidermis causing a vesicular rash with fever. These vesicles crust in the subsequent 7 - 
10 days and the patient is no longer considered infectious after the lesions have crusted. In some cases, the VZV virus infects the neuronal tissue and moves anterogradely and infecting the dorsal root ganglion. Any acute decrease in the cell-mediated immunity can cause the viral reactivation and retrograde spread of the VZV to the terminal ends of the sensory nerves causing a vesicular rash in that particular dermatomal distribution. Scarring and hypopigmentation may occur at the site of the lesions and they may usually take weeks to months to completely resolve. The common complication which occurs in approximately $10 \%$ of the cases is postherpetic neuralgia which is persistent pain at the site of the lesions which can be up to 90 days after the resolution of the lesions. Rowbotham et al. (1998; Kupila et al., 2006) Another common complication is a superimposed bacterial infection at the site of the herpetic vesicular lesions.

VZV infection usually involves the spinal dermatomal distribution but it is not always the case. It may also involve cranial nerve distribution. If it involves the ophthalmic division of the trigeminal nerve it can cause Herpes Zoster Ophthalmicus. Ramsay Hunt Syndrome (Herpes Zoster Oticus) is a classic example in which there is a reactivation of VZV which had remained dormant in geniculate ganglion of the facial nerve upon acute deficiency in cell-mediated immunity.

Very rarely VZV can involve the nerves supplying the meninges of the brain causing aseptic meningitis. Aseptic meningitis is defined as meningitis with negative bacterial cultures in the blood and CSF. In a Finnish study 144 cases of aseptic meningitis were analyzed with PCR of CSF. (Kupila et al., 2006) $8 \%$ of the cases had VZV PCR positive indicating VZV as the cause of meningitis. The most common etiology reported for VZV meningitis is a secondary reactivation per literature review. Another possible etiology is the hematological spread of virus causing neuronal tissue infection in the meninges. The predominant symptoms of VZV meningitis are headache, high fevers and vesicular rash. However other associated symptoms reported are photophobia, phonophobia, neck pain and rigidity, positive Kernig's and Brudzinski's sign. In about 33$60 \%$ of the patients with VZV meningitis, there is no associated rash but, in most cases, there is an associated fever. Yoshikawa et al. (2001; Rowbotham et al., 1998; Kupila et al., 2006) Very few patients don't have a rash at the time of appearance of symptoms of meningitis but do develop a rash 3-5 days later. Extremely rarely some patients may develop a VZV meningitis without any rash or fever as in our patient. However, most of the cases reported of VZV meningitis without a rash had a history of Herpes Zoster infection in the past indicating a possible secondary reactivation as the likely etiology. In our case, the patient did not have any history of Shingles in the past indicating meningitis as a primary infection.
The current gold standard of diagnosis for VZV meningitis is Cerebrospinal fluid PCR due to greater than $95 \%$ sensitivity and high specificity as well $<1$ day needed to get the results of the test. Also, there is no cross-reactivity between different viruses when they were tested on CSF PCR. Harbecke et al. (2009) The CSF analysis on our patient showed lymphocytic pleocytosis, with normal glucose and elevated protein indicating a purely viral aseptic meningitis. Fadhel et al. (2019) reported a similar case of an immunocompetent patient with meningitis with lymphocytic pleocytosis, decreased blood glucose in the CSF analysis and positive VZV PCR in CSF and negative bacterial cultures. Fadhel et al. (2019) It raises the question if it was initially a bacterial infection that improved with intravenous antibiotics, with secondary VZV reactivation showing a positive VZV PCR in CSF. However, in our case CSF analysis was very typical for viral meningitis. Spernovasilis et al. (2018; Habib et al., 2009; Pasedag et al., 2014) have reported similar cases of VZV meningitis in immunocompetent patients with hypoglycorrhachia in CSF and without rash and fever. Grahn and Studahl (2015; Becerra et al., 2013; Habib et al., 2009; Pasedag et al., 2014; Spernovasilis et al., 2018) However, in our patient CSF glucose was slightly increased possible secondary to the steroids he received for the treatment of misdiagnosed sinusitis. The $\mathrm{CSF} / \mathrm{Blood}$ glucose ratio being normal suggests a purely viral etiology in our case.

The current standard of care for VZV meningitis is IV Acyclovir $1 \mathrm{~g}$ every $8 \mathrm{~h}$. It is switched to oral Valacyclovir $800 \mathrm{mg}$ every 6 hours pending clinical improvement. Most patients are given a total of 10-14 days of treatment. In certain cases, the duration of the treatment may be extended to a total of 21 days. Early initiation of IV acyclovir in the patients suspected to have meningitis has shown to significantly reduced morbidity. Habib et al. (2009; Pasedag et al., 2014; Spernovasilis et al., 2018; Tyring et al., 2000) Due to the national shortage of IV Acyclovir in the United States at the time of the presentation, our patient was treated initially with IV Ganciclovir and was switched to oral Valacyclovir after clinical improvement. The patient was followed up 2 weeks later in the infectious disease clinic and no neurological sequelae of VZV infection were noted (Tyring et al., 2000).

Our case is unique in the sense that the patient was immunocompetent, had no history of VZV primary infection in the past, had no rash or fever at the time of presentation and no rash development 5 days after the presentation, did not have hypoglycorrhachia on CSF analysis and no neurological symptoms. The purpose of this case report is to indicate that VZV infection can present as meningitis even in an immunocompetent patient. Current CDC (Centre for Disease Control) guidelines suggest airborne precautions in VZV shingles 
until the lesions crust. However, there are currently no guidelines about air borne precautions in VZV meningitis. This will need further research. The author suggests observing the airborne precautions for health care workers treating VZV meningitis patients.

\section{Learning Points/Take Home Messages 3-5 Bullet Points}

1. VZV Zoster Virus is a rare cause of aseptic meningitis. It should be included in the differential for any case of a patient presenting with meningitis

2. Rash and fever are not always present with VZV meningitis making it a diagnostic challenge. 3 . Early initiation of IV acyclovir has shown to reduce morbidity in the patients. Hence IV acyclovir should be started early in any patient in whom meningitis is suspected

3. Even if a health care worker is vaccinated against VZV and is immunocompetent, airborne and contact precautions should be observed

\section{Acknowledgement}

The author acknowledges the support provided by Michigan State University Internal Medicine Residency program for the completion of this manuscript.

\section{Ethics}

The author does not see any ethical issues that may arise after the publication of this manuscript.

\section{References}

Antonelli, M.A., L.W. Moreland and J.E. Brick, 1991. Herpes zoster in patients with rheumatoid arthritis treated with weekly, low-dose methotrexate. Am. J. Med., 90: 295-295.

DOI: 10.1016/0002-9343(91)90568-I

Becerra, J.C.L., R. Sieber, G. Martinetti, S.T. Costa and P. Meylan, 2013. Infection of the central nervous system caused by Varicella Zoster Virus reactivation: A retrospective case series study. Int. J. Infect. Dis., 17: 529-534. DOI: 10.1016/j.ijid.2013.01.031

Chen, S.Y., J.A. Suaya, Q. Li, D. Misurski and S. Burstin et al., 2014. Incidence of herpes zoster in patients with altered immune function. Infection, 42: 325-334. DOI: 10.1007/s15010-013-0550-8

Fadhel, M., N. Campbell, S. Patel, A. Mushtaq and Lito Fune et al., 2019. Varicella zoster meningitis with hypoglycorrhachia on Cerebrospinal Fluid (CSF) analysis in a Young Immunocompetent Host without a Rash. Am. J. Case Rep., 20: 701-704.

DOI: 10.12659/AJCR.915300
Grahn, A. and M. Studahl, 2015. Varicella-zoster virus infections of the central nervous system-prognosis, diagnostics and treatment. J. Infect., 71: 281-293. DOI: $10.1016 /$ j.jinf.2015.06.004

Habib, A.A., D. Gilden, D.S. Schmid and J.E. Safadieh, 2009. Varicella Zoster Virus meningitis with hypoglycorrhachia in the absence of rash in an immunocompetent woman. J. Neurovirol., 15: 206-208. DOI: 10.1080/13550280902725550

Harbecke, R., M.N., Oxman, B.A. Arnold, C. Ip and G.R. Johnson et al., 2009. A real-time PCR assay to identify and discriminate among wild-type and vaccine strains of varicella-zoster virus and herpes simplex virus in clinical specimens and comparison with the clinical diagnoses. J. Med. Virol., 81: 1310-1322. DOI: 10.1002/jmv.21506

Harpaz, R., I.R. Ortega-Sanchez and J.F. Seward, 2008. Advisory Committee on Immunization Practices (ACIP) Centers for Disease Control and Prevention (CDC). Prevention of herpes zoster: Recommendations of the Advisory Committee on Immunization Practices (ACIP). MMWR Recomm. Rep.

Josephson, A. and M.E. Gombert, 1988. Airborne transmission of nosocomial varicella from localized zoster. J. Infect. Dis., 158: 238-241.

DOI: 10.1093/infdis/158.1.238

Kupila, L., T. Vuorinen, R. Vainionpää, R.J. Marttila and P. Kotilainen, 2006. Etiology of aseptic meningitis and encephalitis in an adult population. Neurology, 66: 75-75.

DOI: 10.1212/01.wnl.0000191407.81333.00

Miller, A.E., 1980. Selective decline in cellular immune response to varicella-zoster in the elderly. Neurology, 30: 582-582. DOI: 10.1212/WNL.30.6.582

Opstelten, W., G.A. Van Essen, F. Schellevis, T.J.M. Verheij and K.G.M. Moons et al., 2006. Gender as an independent risk factor for herpes zoster: A population-based prospective study. Ann. Epidemiol., 16: 692-695.

DOI: $10.1016 /$ j.annepidem.2005.12.002

Pasedag, T., K. Weissenborn, U. Wurster and M. Stangel et al., 2014. Varicella Zoster Virus meningitis in a young immunocompetent adult without rash: A misleading clinical presentation. Case Rep. Neurol. Med.

Rowbotham, M., N. Harden and B. Stacey, 1998. Gabapentin for the treatment of postherpetic neuralgia: A randomized controlled trial. JAMA, 280: 1837-1842. DOI: 10.1001/jama.280.21.1837

Schmader, K., L.K. George, B.M. Burchett, C.F. Pieper and J.D. Hamilton, 1995. Racial differences in the occurrence of herpes zoster. J. Infect. Dis., 171: 701-704. DOI: 10.1093/infdis/171.3.701 
Spernovasilis, N., A. Milioni, I. Gialamas, E. Kokorakis and G. Fanti, 2018. Varicella-zoster virus meningitis with hypoglycorrhachia in a young immunocompetent adult without rash: A case report and literature review. IDCases, 12: 104-106. DOI: $10.1016 /$ j.idcr.2018.04.008

Thomas, S.L., J.G. Wheeler and A.J. Hall, 2004. Casecontrol study of the effect of mechanical trauma on the risk of herpes zoster. BMJ, 328: 439-439. DOI: 10.1136/bmj.37991.511829.F7

Tyring, S.K., K.R. Beutner and B.A. Tucker, 2000. Antiviral therapy for herpes zoster: Randomized, controlled clinical trial of valacyclovir and famciclovir therapy in immunocompetent patients 50 years and older. Arch. Fam. Med., 9: 863-863. DOI: 10.1001/archfami.9.9.863
Weinberg, A., A.A. Lazar, G.O. Zerbe, A.R. Hayward and I.S.F. Chan et al., 2010. Influence of age and nature of primary infection on varicella-zoster virusspecific cell-mediated immune responses. J. Infect. Dis., 201: 1024-1030. DOI: 10.1086/651199

Yawn, B.P., P. Saddier, P.C. Wollan, J.L.S. Sauver and M.J. Kurland et al., 2007. A population-based study of the incidence and complication rates of herpes zoster before zoster vaccine introduction. Mayo Clin. Proc., 82: 1341-1349. DOI: 10.4065/82.11.1341

Yoshikawa, T., M. Ihira, K. Suzuki, S. Suga and A. Tomitaka et al., 2001. Rapid contamination of the environments with varicella-zoster virus DNA from a patient with herpes zoster. J. Med. Virol., 63: 64-66. DOI: 10.1002/10969071(200101)63:1<64::AID-JMV1009>3.0.CO;2-6 